\title{
A Shannon entropy approach to the temporal evolution of SEP energy spectrum
}

\author{
M. Laurenza ${ }^{1}$, G. Consolini ${ }^{1}$, M. Storini ${ }^{1}$, and A. Damiani ${ }^{1, *}$ \\ ${ }^{1}$ INAF/IFSI-Roma, Via del Fosso del Cavaliere 100, 00133, Roma \\ * presently at: Physics Department, University of Santiago de Chile, Santiago, Chile \\ Correspondence to: M. Laurenza (monica.laurenza@ifsi-roma.inaf.it)
}

Received: 21 October 2010 - Revised: 10 March 2011 - Accepted: 12 April 2011 - Published: 28 February 2012

\begin{abstract}
The kinetic energy spectra of solar energetic particle (SEP) events contain information on the particle acceleration mechanisms. A novel approach is proposed to investigate the time evolution of the spectral shape for the 20 February 2002 SEP event. Proton differential fluxes recorded by the ERNE instrument aboard SOHO, in the energy range $1.67-112 \mathrm{MeV}$, are analyzed. The solar source is associated with this SEP event by studying solar and interplanetary conditions during the considered time period. The energy spectrum evolution is studied by evaluating the time history of Shannon's differential entropy derived from the SEP fluxes. Our findings suggest that particle acceleration in the considered event is produced by a perpendicular shock in the solar corona. Moreover, dropouts in the particle flux are clearly identified up to energies of at least $\sim 10 \mathrm{MeV}$.
\end{abstract}

\section{Introduction}

Solar energetic particles (SEPs) are thought to be accelerated by both solar flares and shocks driven by coronal mass ejections (CMEs). In particular, according to the two-class paradigm (Reames, 1999), flares are responsible for particle acceleration in impulsive SEP events, whereas CME driven shock waves accelerate particles within a large volume of the solar corona and interplanetary space during large gradual SEP events (e.g. Lario et al, 1998; Lehtinen et al., 2008; Verkhoglyadova et al., 2010). In the case of gradual SEP events, the spectral shape is related to the characteristics of the CME-driven shock; it can be also highly dynamic as a consequence of propagation effects. On the other hand, impulsive SEP events are thought to be rapidly accelerated in solar flares and to propagate fast enough that adiabatic energy changes can be neglected; hence their spectrum should represent the source spectrum (e.g. Dröge, 2000).

Impulsive and gradual SEP events are identified from energetic particle measurements also in terms of their abun- dances, ionization states, duration and longitude distributions of the associated solar sources. Nevertheless, a more complex scenario was underlined by Kocharov and Torsti (2002) in the case of hybrid SEP events, supposedly consisting of a mixture of the flare-accelerated particles population and the CME related one. At present, the principal acceleration mechanism for $>10 \mathrm{MeV}$ protons is still under debate. The variability observed in the spectral features and elemental composition from one event to another, has been interpreted by Tylka et al. (2005) in terms of shock geometry and seed population. On the other hand, Cane et al. $(2002,2010)$ have invoked a post-CME (flare) reconnection process. A revised classification scheme has recently been proposed by Cliver (2009): the old impulsive and gradual classes are referred to as "flare" and "shock" classes, respectively. Moreover, the shock class is subdivided into two groups, according to the shock geometry, namely quasi-perpendicular and quasiparallel shocks, acting on flare and coronal/solar wind seed particles, respectively.

Nearly all previous studies (see, for instance, Cohen et al., 2005; Mewaldt et al., 2005) examined event-integrated spectra in order to understand the different processes involved in the event, although it is important to investigate how the spectral characteristics evolve during the event (Tylka et al., 2000).

In this paper, we present a preliminary study for the spectrum evolution of the 20 February 2002 SEP event, by following a novel approach, based on information theory. The paper is organized as follows. We describe the method in Sect. 2 and its application to the 20 February 2002 SEP event in Sect. 3.

\section{Information entropy definitions}

One of the major results of information theory is the Shannon entropy (also named information entropy) (Shannon, 1948), 


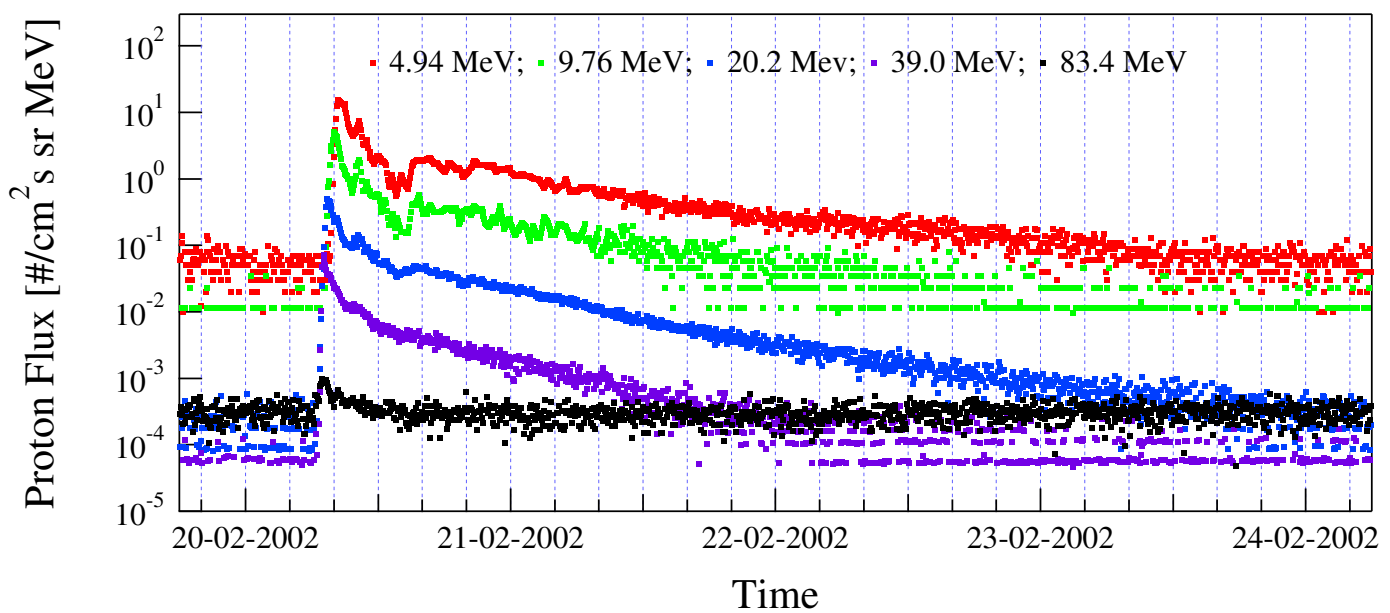

Fig. 1. Temporal behavior of the proton differential flux as recorded in five energy channels (geometric mean energy reported in the legend) by SOHO/ERNE for the 20 February 2002 SEP event.

a quantity capable of estimating the degree of uncertainty and/or the information contained in a sequence of characters or in a signal.

Let us suppose that a discrete variable $X$ can assume $N$ different values $x_{1}, x_{2}, \ldots ., x_{N}$, and indicate with $p_{i}$ the probability of finding the variable $X$ to be $x_{i}$; then the Shannon entropy $S_{X}$ is defined by the probabilities $p_{i}$ as

$S_{X}=-k \sum_{i=1}^{N} p_{i} \ln p_{i}$,

where $k$ is a constant, generally assumed $k=1$.

From Eq. (1) it follows that such a quantity takes its maximum value, $S_{\max }=\ln N$, for uniform probability distributions $\left(p_{i}=1 / N \forall i\right)$, while it is zero in the case of delta-like distributions $\left(p_{i}=0 \forall i \neq j\right.$ and $p_{j}=1$, where $j \in[1, N]$ ). All the intermediate situations would provide a value of this quantity in the range $[0, \ln N]$, thus, resulting sensitive to the changes of the shape of the probability distribution $p_{i}$. In other words, increasing values of the Shannon entropy correspond to an increasing degree of the uncertainty.

Although the Shannon entropy has been introduced for discrete variables, it is possible to generalize the expression of Eq. (1) to the case of continuous variables $(x)$, where the probability $p_{i}$ is replaced by a probability density $p(x)$ with $x \in \Omega$, where $\Omega$ is the range of variability of the variable $x$ :

$S_{X}=-k \int_{\Omega} p(x) \ln p(x) d x$

This quantity is generally referred to as the Shannon differential entropy. Due to sensitivity of the information entropy $S_{X}$ to the distribution shape we, here, attempt an approach to the evolution of the differential flux spectrum during a SEP event, in order to quantify the changes of the spectrum shape with time. Because the spectrum (although obtained by using discrete energy channels of different width) is a continuous function of the energy, we use the definition of Eq. (2) to evaluate the information entropy. To do this, it is first of all necessary to define an appropriate probability density measure $p(E)$. Thus, if $\delta F(E)$ is the differential flux measured at the energy $E$, we can introduce an associated probability density measure $p(E)$ as

$p(E)=\frac{\delta F(E)}{\int_{\Omega(E)} \delta F(E) d E}$,

where $\Omega(E)$ is the interval of energies considered. We note that $p(E)$ has all the required properties to be a probability measure, i.e. $i) p(E) \geq 0$ and $i i) \int_{\Omega(E)} p(E) d E=1$.

When the above approach is applied to the evolution of the differential flux spectra an increase and/or decrease of Shannon's differential entropy can provide information on the changes of the spectrum shape in the considered energy interval due to additional high energy particle contributions with respect to the background condition. However, it is important to remark that the single Shannon's differential entropy value it is not sufficient to get a complete description of the spectral shape. Indeed, it is required both the knowledge of the background spectrum shape and the differential entropy value. We will discuss this point in the next Section.

It is important to remark that in spite of its definition, the Shannon's differential entropy, as applied here, has not to be confused with Boltzmann thermodynamic statistical entropy. As a matter of fact, we use information entropy only as a proxy of changes of spectrum shape in a limited range of energies. 


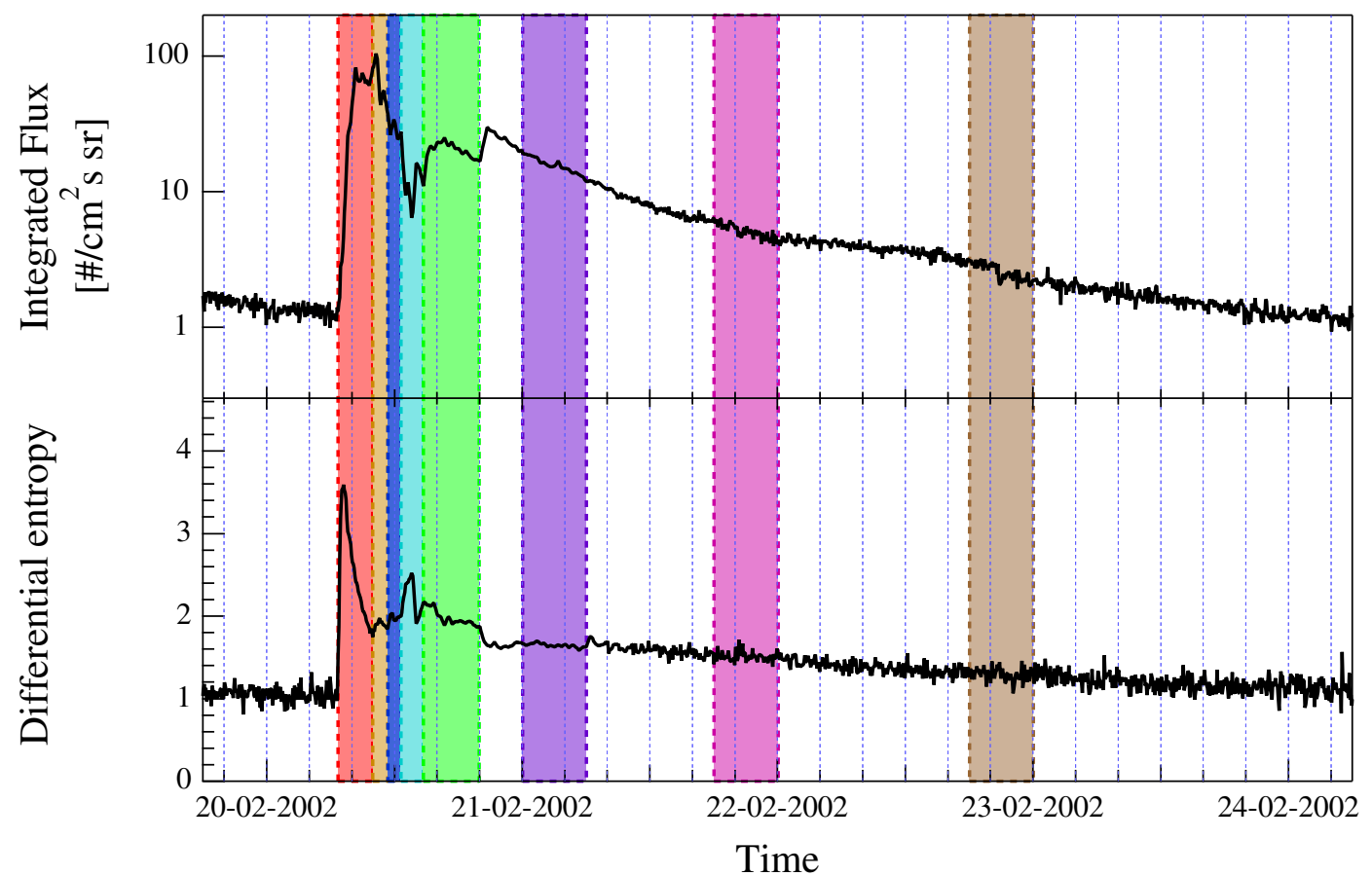

Fig. 2. Energy integrated flux (upper panel) and Shannon differential entropy computed for the 20 February 2002 SEP event (lower panel). Colored bands indicate time intervals during which the different spectra of Fig. 3 have been derived.

Table 1. 20 February 2002 SEP event associated solar source and interplanetary conditions.

\begin{tabular}{lllllll}
\hline Active Region & Location & X-ray Flare & CME & Type II & Type IV & Shock passage \\
\hline \multirow{2}{*}{9825} & ${\mathrm{~N} 12^{\circ} \mathrm{W} 72^{\circ}}$ & class: M5.7 & halo & yes & yes & no \\
& & peak time: 06:12 UT & time: 06:30 UT & & & \\
& e-folding time:00:04h & speed: $952 \mathrm{~km} / \mathrm{s}$ & & & \\
\hline
\end{tabular}

\section{Spectrum evolution}

We used 5-min averages of the proton flux recorded by the ERNE instrument onboard SOHO, at 58 energy channels from 1.67 to $112 \mathrm{MeV}^{1}$. Figure 1 shows the temporal behavior of the proton differential flux for five energy channels as observed during the 20 February 2002 SEP event. As expected, the largest fluxes are observed at the lowest energies; a velocity dispersion is evident in the prompt phase of the event, associated with the arrival of high energy particles first and lower energy ones later; a rapid decrease at the lowest energies is observed in the time interval from 8:00 UT to 12:00 UT. The latter feature is also apparent in the flux integrated over the whole energy range as shown in the upper panel of Fig. 2. This event can be classified as an impulsive one (Chollat and Giacalone, 2008), although it also shows gradual characteristics as it can be seen in Table 1 (e.g. long duration, type II and IV radio emissions).

\footnotetext{
${ }^{1}$ http://www.srl.utu.fi/erne_data/main_english.html
}

The behaviour of the Shannon differential entropy, shown in the lower panel of Fig. 2, changes throughout all the event, reflecting the continuous evolution of the spectral shape, as depicted in Fig. 3. Because of the presence of a power lawlike background spectrum, the emergence of another contribution at high energies due to the particle injection tends to reduce the spectrum slope. This flattening reflects onto an increase of the Shannon differential entropy value. Hence, during the considered event prompt phase, the fast rise of the Shannon differential entropy indicates that the spectrum is flattening, i.e. the contribution of high energy particles is increasing. Indeed, the lack of low energy particles is due to the velocity dispersion effect, as it can be seen by comparing the two spectra relative to the prompt phase (red and ocher circles in Fig. 3). This effect vanishes during the Shannon differential entropy decay (before 09:57 UT), as also indicated by the constancy of the energy integrated flux (upper panel of Fig. 2) during the same period.

Soon after the prompt phase of the event, the Shannon differential entropy (lower panel of Fig. 2) shows an additional 


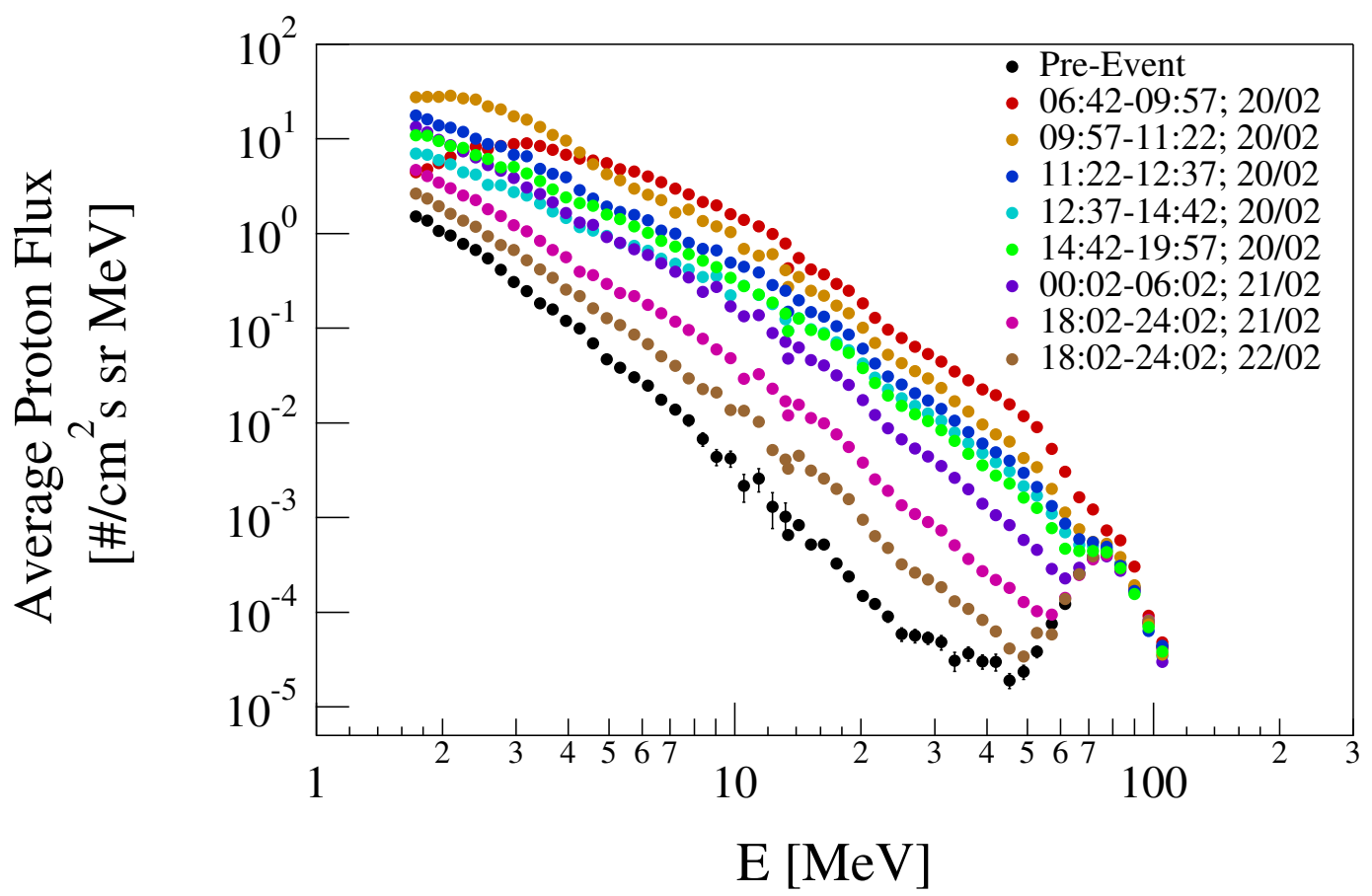

Fig. 3. Spectrum evolution for the 20 February 2002 SEP event. Colors of spectra are coded according to the different time intervals shown in Fig. 2.

peak (cyan stripe), which may be read as the occurrence of a new process, besides the main one producing the event. In fact, the particle distribution is becoming again more entropic with a flatter spectrum and a greater contribution of high energies. Nevertheless, a new particle injection during the SEP event can be excluded because no velocity dispersion effect is apparent in the particle flux (note also that the second peak has a very sharp decay as opposed to the first one). Correspondingly, the spectra computed before, during and after the peak (time periods are those indicated by blue, cyan and green stripes in Fig. 2, respectively) are displayed in Fig. 4. A noticeable change in the spectral shape is observed: during the peak period the spectrum flattens at low energies ( $E<10 \mathrm{MeV}$; see dashed line in Fig. 4); it becomes sharper again soon after the peak. This is consistent with a temporary lack of low energy particles, which soon recovers. This behavior can be related to the presence of dropouts (Mazur et al., 2000; Chollat and Giacalone, 2008). Later, during the decay phase of the event (i.e. when the total number of particles is decreasing), the Shannon differential entropy as well as the computed spectra show a more gradual evolution, during which the relative contribution of low and high energies continuously changes. It follows that an event integrated spectrum might not be representative of the acceleration mechanisms responsible for the event.

In order to have information on the acceleration process at the Sun, we computed the spectrum during the time period between 09:57 - 12:37 UT, i.e. still in the initial phase of the

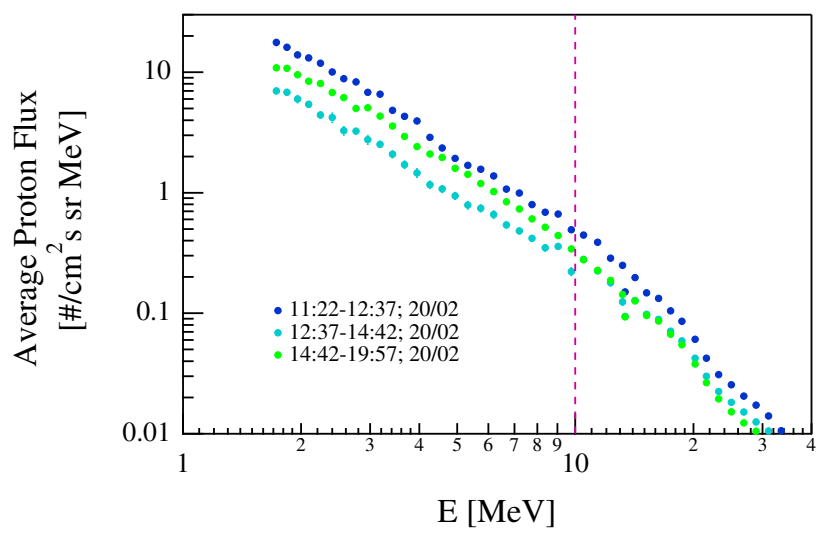

Fig. 4. A zoom of the spectrum evolution for the 20 February 2002 SEP event during the time interval 11:22 UT - 19:57 UT. The vertical violet dashed-line indicates the energy threshold above which the effects of drop-out are not present.

event (thus related to the acceleration at the solar source), but after the end of the velocity dispersion effect. During this period the Shannon differential entropy is relatively constant between the two main peaks. Figure 5 illustrates the obtained spectrum (red dots) along with the average spectrum (blue dots) computed in a different time interval at the beginning of the event decay phase, when the Shannon differential entropy is constant (violet stripes in Fig. 2). Both spectra are fitted with the following forms, expected from shock acceleration, 
Table 2. Fit parameters for the 20 February 2002 SEP event.

\begin{tabular}{lll}
\hline Time (UT) & Ellison-Ramaty, 1985 & Tylka et al., 2005 \\
\hline $09: 57-12: 37 ; 20 / 02$ & $K_{0}=100 \pm 10$ & $C=85 \pm 10$ \\
& $\gamma=1.94 \pm 0.08$ & $\gamma_{a}=1.7 \pm 0.2 ; \gamma_{b}=3.5 \pm 0.2$ \\
& $E_{0}=16 \pm 2$ & $E_{*}=12 \pm 2$ \\
$20: 52-05: 57 ; 20-21 / 02$ & $K_{0}=64 \pm 7$ & $C=58 \pm 8$ \\
& $\gamma=2.33 \pm 0.07 ;$ & $\gamma_{a}=2.2 \pm 0.1 ; \gamma_{b}=3.7 \pm 0.2 ;$ \\
& $E_{0}=19 \pm 2$ & $E_{*}=14 \pm 3$ \\
\hline
\end{tabular}

proposed by Ellison and Ramaty (1985) [Eq. (4)] and Tylka et al. (2005) [Eq. (5)], respectively:

$$
\frac{d J}{d E}=K_{0} E^{-\gamma} \exp \left(-\frac{E}{E_{0}}\right)
$$

$\frac{d J}{d E}=\left\{\begin{array}{cc}C E^{-\gamma_{a}} e^{-\frac{E}{E_{0}}} & \text { for } E \leq E_{*} \\ C E^{-\gamma_{b}}\left[E_{*}^{\left(\gamma_{a}-\gamma_{b}\right)} e^{\left(\gamma_{a}-\gamma_{b}\right)}\right] & \text { for } E \geq E_{*}\end{array}\right.$

where $J$ is the intensity, $E$ is kinetic energy and $E_{*}=\left(\gamma_{b}-\right.$ $\left.\gamma_{a}\right) E_{0}$. Parameters $\gamma$ and $E_{0}$ in Eq. (4) are related to the shock compression ratio and to the diffusion coefficient that controls the escape from the near shock region. In Eq. (5) $\gamma_{a}$ is the low energy power law slope and $\gamma_{b}$ is the high energy power law slope. The function is identical to the EllisonRamaty form below the transition energy, $\left(\gamma_{b}-\gamma_{a}\right) E_{0}$. At higher energies, the function makes a smooth transition to a second power law. This spectral form gives an improved fit to the high energy spectra, having a lower reduced $\chi$ square than the Ellison-Ramaty form.

The parameters of the fits are summarized in Table 2. We point out that fits were performed over points at energies $<52.7 \mathrm{MeV}$, as the high instrumental background at higher energies might have compromised their values. It is apparent that at energies $<14 \mathrm{MeV}$ ( $>14 \mathrm{MeV}$ ) the spectrum is harder (softer) than that computed in the prompt phase. Hence, assuming shock acceleration, the observed softening of the spectrum should be indicative of an increase in the diffusion coefficient (Zank et al., 2000), resulting in a decreasing ability of a shock to contain and further accelerate particles at energy $>14 \mathrm{MeV}$ (rollover energy).

\section{Summary and Conclusions}

The spectrum evolution in time has been analyzed for the 20 February 2002 SEP event. We identified significant changes in the spectral shape by taking advantage of the Shannon differential entropy as a single parameter, combined with inspection of the actual spectrum. Main results can be summarized as follows:

- The Shannon differential entropy for the 20 February 2002 event shows two main peaks, both indicating an abrupt

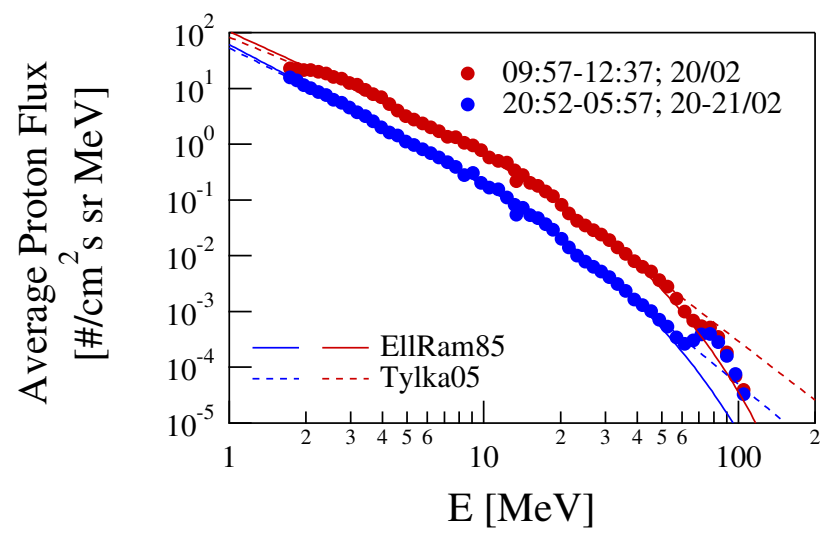

Fig. 5. Time averaged spectrum for the 20 February 2002 SEP event for two different time intervals (see the legend). Solid and dashed lines are best fits using the two functional forms of Eq. (4) and (5), respectively.

change in the particle distribution. The first one occurs at the event onset and reflects the spectrum variations due to velocity dispersion. In addition, by examining the energy spectrum evolution when the second peak occurs, dropouts are detected up to at least $\sim 10 \mathrm{MeV}$. To our knowledge, this is the first evidence of a dropouts event at such energies and, in particular, using particle data recorded by ERNE aboard SOHO.

- The study of the event solar source indicates the presence of a coronal shock, which propagates in the interplanetary medium without impacting on the Earth. Accordingly, fit of the event spectrum, during the prompt phase but when the velocity dispersion is over, is consistent with a shock acceleration mechanism (a double exponential with break point at $E=(12 \pm 1) \mathrm{MeV})$. Nevertheless, the SEP event presents impulsive characteristics. These features suggest that acceleration can be produced by a quasi-perpendicular shock in the solar corona, although a direct contribution from the flare cannot be excluded.

- The Shannon differential entropy exhibits a gradual but continuous change in the decay phase of the considered event, indicating a slowly evolution of the event spectrum (i.e a non stationary shape), particularly at lower energies. A reduced acceleration capability of the evolving shock is sug- 
gested for energies $>14 \mathrm{MeV}$.

The principal merit of the application of the Shannon differential entropy in the investigation of the SEP spectrum evolution seems to be the high sensitivity in detecting changes in the spectral shape due to additional particle contributions (e.g. new injections, dropouts). Indeed, being computed on the basis of normalized quantities $(p(E) d E)$ the Shannon differential entropy is not affected by particle density variations. Further work is necessary to explore the application of information approach on a wide statistics of SEP events.

Acknowledgements. We acknowledge the SOHO/ERNE instrument teams as well as corresponding data and science centers for providing data used in this research. MS thanks for the partial financial support the Local Organising Committee of ECRS 2010 (Turku, Finland, 3-6 August 2010), where part of this work was presented. This paper is supported by the Italian Space Agency (contract ASI/INAF n. I/022/10/0) for the BepiColombo Mission.

Edited by: B. Heber

Reviewed by: K. Scherer and another anonymous referee

\section{References}

Cane, H. V., Erickson, W. C., and Prestage, N. P.: Solar flares, type III radio bursts, coronal mass ejections, and energetic particles, J. Geophys. Res., 107, 1315, doi:10.1029/2001JA000320, 2002.

Cane, H. V., Richardson, I. G., and von Rosenvinge, T. T.: A study of solar energetic particle events of $1997-2006$ : Their composition and associations, J. Geophys. Res., 115, A08101, doi:10.1029/2009JA014848, 2010.

Chollat, E. E. and Giacalone, J.: Multispacecraft analysis of energetic ion flux dropouts, Astrophys. J., 688, 1368-1373, 2008.

Cliver, E. W.: A revised classification scheme for solar energetic particle events, Centr. Eur. Bull., 33, 253-270, 2009.

Cohen, C. M. S., Stone, E. C., Mewaldt, R. A., Leske, R. A., Cummings, A. C., Mason, G. M., Desai, M. I., von Rosenvinge, T. T., and Wiedenbeck, M. E.: Heavy ion abundances and spectra from the large solar energetic particle events of October-November 2003, J. Geophys. Res., 110, A09S16, doi:10.1029/2005JA011004, 2005.

Dröge, W.: Solar Flare Proton Spectra, High Energy Solar Physics Workshop - Anticipating HESSI, ASP Conf. Series, 206, edited by: R. Ramaty and N. Mandzhavidze, 191, 2000.
Ellison, D. C. and Ramaty, R.: Shock acceleration of electrons and ions in solar flares, Astrophys. J., 298, 400-408, 1985.

Kocharov, L. and Torsti, J.: Hybrid solar energetic particle events observed on board Soho, Sol. Phys., 207, 149-157, 2002.

Lario, D., Sanahuja, B., and Heras, A. M.: Energetic particle events: efficiency of interplanetary shocks as $50 \mathrm{keV}<E<100 \mathrm{MeV}$ proton accelerators, Astrophys. J., 509, 415-434, 1998.

Lehtinen, N. J., Pohjolainen, S., Huttunen-Heikinmaa, K., Vainio, R., Valtonen, E., and Hillaris, A. E.: Sources of SEP Acceleration during a Flare - CME Event, Sol. Phys., 247, 151-169, 2008.

Mazur, J. E., Mason, G. M., Dwyer, J. R., Giacalone, J., Jokipii, J. R., and Stone, E. C.: Interplanetary magnetic field line mixing deduced from impulsive solar flare particles, Astrophys. J., 532, 79-82, 2000.

Mewaldt, R. A., Cohen, C. M. S., Labrador, A. W., Leske, R. A., Mason, G. M., Desai, M. I., Looper, M. D., Mazur, J. E., Selesnick, R. S., and Haggerty, D. K.: Proton, helium, and electron spectra during the large solar particle events of October-November 2003, J. Geophys. Res., 110, A09S18, doi:10.1029/2005JA011038, 2005.

Reames, D. V.: Particle acceleration at the Sun and in the heliosphere, Space Sci. Rev., 90, 413-491, 1999.

Shannon, C. E.: A Mathematical Theory of Communication, Bell Syst. Tech. J., 27, 379-423, 1948.

Tylka, A. J., Boberg, P. R., McGuire, R. E., Ng, C. K., and Reames, D. V.: Temporal evolution in the spectra of gradual solar energetic particle events, in: Acceleration and transport of energetic particles observed in the heliosphere: ACE 2000 Symp., AIP Conf. Proc., 528, 147-52, 2000.

Tylka, A. J., Cohen, C. M. S., Dietrich, W. F., Lee, M. A., Maclennan, C. G., Mewaldt, R. A., Ng, C. K., and Reames, D. V.: Shock geometry, seed populations, and the origin of variable elemental composition at high energies in large gradual solar particle events, Astrophys. J., 625, 474-495, 2005.

Verkhoglyadova, O. P., Li, G., Zank, G. P., Hu, Q., Cohen, C. M. S., Mewaldt, R. A., Mason, G. M., Haggerty, D. K., von Rosenvinge, T. T., and Looper, M. D.: Understanding large SEP events with the PATH code: Modeling of the 13 December 2006 SEP event, J. Geophys. Res., 115, A12103, doi:10.1029/2010JA015615, 2010.

Zank, G. P., Rice, W. K. M., and Wu, C. C.: Particle acceleration and coronal mass ejection driven shock: a theoretical model, J. Geophys. Res., 105, 25079-25095, 2000. 